\title{
La représentation des poétesses dans la presse de la fin de la Belle Époque (1908-1914)
}

\author{
Wendy PrIN-CONTI \\ Université Sorbonne Nouvelle - Paris 3 \\ wendy.princonti@gmail.com \\ https://orcid.org/oooo-0002-2662-4802
}

\section{Resumen}

El objetivo de este artículo es observar y comparar la manera con la cual los poetas y las poetisas de la Belle Époque fueron representados en la prensa francesa de la época. La investigación se centra en los retratos fotográficos publicados entre 1908 y 1914 en Les Annales politiques et littéraires, Femina y Comoedia. Se tratará de mostrar de qué manera los años previos a la primera guerra mundial constituyeron un momento crucial para el acceso de las mujeres autoras a una forma de reconocimiento público.

Palabras clave: Belle Époque, Fotografía, Poetisas, Prensa, Medios.

\section{Résumé}

L'objet de la présente étude est d'observer et de comparer la manière dont les poètes et poétesses français de la fin de la Belle Époque ont été représentés dans la presse nationale du temps. Notre enquête se concentre sur les portraits photographiques publiés entre 1908 et 1914 dans Les Annales politiques et littéraires, Femina et Comodia. Il s'agira de montrer en quoi les années d'avant-guerre constituent un moment charnière dans l'accession des femmes auteures à une forme de reconnaissance publique.

Mots-clés : Belle Époque, Photographie, Poétesses, Presse, Médias.

\section{Abstract}

This work aims at observing and comparing the image of French poets and poetess given by the national press during the late Belle Époque. More precisely, we study the photographs published by Les Annales politiques et littéraires, Femina and Comoedia, between 1908 and 1914. We shall prove that female writers obtained public recognition immediately before the First World War.

Keywords: Belle Époque, Photography, Poetess, Press, Media.

${ }^{*}$ Artículo recibido el 16/06/2021, aceptado el 19/10/2021. 


\section{Introduction}

L'objet de la présente étude est d'observer et de comparer la manière dont les poètes et poétesses de la fin de la Belle Époque ont été représentés dans la presse du temps. Il s'agira de montrer en quoi les années d'avantguerre constituent un moment charnière dans l'accession des femmes auteures à une forme de reconnaissance publique.

Notre corpus, tentaculaire, sera circonscrit selon trois critères. Nous choisissons premièrement de borner nos observations aux années 19081914. La comparaison entre poètes et poétesses ne devient en effet opérante que dans les toutes premières années du vingtième siècle. Auparavant, les qualités littéraires des femmes sont encore largement minorées (Holmes et Tarr, 2006 ; Izquierdo, 2009 ; Le Guennec et Zmelty, 2013). Partant, leur image n'est pas ou que très peu utilisée par la presse. Le tournant que constituent les années 1907-1908 peut ainsi être considéré comme le terminus a quo de notre étude. À partir de cette date en effet, les comptes rendus consacrés aux femmes poètes cessent d'être systématiquement à charge (PrinConti, à paraître). La parution, quasiment conjointe, de trois recueils contribue à cette évolution : il s'agit de L'Instant éternel d'Hélène Picard, des Éblouissements d'Anna de Noailles et de Jeunesse de M" ${ }^{\text {me }}$ Fernand Gregh. Séduits par ces textes qu'ils reconnaissent être de tout premier ordre, d'éminents critiques, à l'image d'Auguste Dorchain, s'emploient dès lors à faire connaître une poésie qu'ils avaient d'abord mésestimée. La déclaration de guerre, qui met brutalement au premier plan une actualité toute politique et militaire, sera, sans grande surprise, notre terminus ad quem.

Outre le critère chronologique, nous prenons le parti de mener notre enquête en concentrant nos regards sur trois journaux et revues sélectionnés selon des considérations sociologiques. Les Annales politiques et littéraires est un hebdomadaire fondé en 1883 , à destination de la bourgeoisie provinciale et coloniale. Cette revue grand public, de vulgarisation culturelle (Pluet-Despatin, Leymarie et Mollier, 2002), permet d'examiner la manière dont le Français moyen reçoit alors l'image des poètes et poétesses. Femina, bi-mensuel créé en 1901, présente quant à lui la particularité de viser un lectorat féminin. Il s'agira de se demander si le choix d'une audience essentiellement féminine a une quelconque incidence sur la manière dont les poétesses sont représentées dans ces pages. Enfin, Comœdia est un quotidien d'information culturelle, fondé au moment du tournant de 1907. Cette publication s'adresse à un public friand de nouveauté littéraire, plus spécialisé que celui des Annales et de Femina. Là encore, il faudra voir si la cible choisie influe, d'une manière ou d'une autre, sur l'image qui est donnée des hommes et femmes poètes du temps.

Pour finir, nous choisissons de nous concentrer, pour l'essentiel, sur la photographie, en excluant volontairement les caricatures et les dessins qui mériteraient une étude à eux seuls. Notre étude porte sur les balbutiements de l'usage de ce medium dans la presse : elle se situe après le « seuil 
technique » (Kalifa, 2001:57) des années 1890 qui voient se développer les procédés photomécaniques, mais avant les années 1920 et la généralisation du procédé offset qui imposera la photographie dans la presse française.

\section{Une tutelle masculine omniprésente (1908-1910)}

Jusqu'en 1910, bien rares sont les poétesses reconnues et représentées pour ce qu'elles sont. Si leurs photographies sont exploitées par la presse, c'est, le plus souvent, en raison de l'activité publique de leur mari. Le cas de Rosemonde Gérard est à ce titre tout à fait éclairant. Petite-fille du général d'Empire Étienne Maurice Gérard, cette descendante de $M^{\text {me }}$ de Genlis a pour parrain Leconte de Lisle. L'auteur des Poèmes antiques encourage très tôt la vocation poétique qu'il pressent chez sa filleule : dès son plus jeune âge en effet, il l'engage à réciter ses vers devant lui. Bien vite, il la présente à José-Maria de Heredia et à Henri de Régnier chez qui elle déclame avec aplomb ses dernières compositions. Ce soutien est tout à fait exceptionnel. Comme le rappelle Gaston Deschamps ${ }^{1}$ :

Leconte de Lisle n'aimait pas, en général, les jeunes filles qui font des vers. Il se méfiait du cahier bleu des pensionnaires. Il décourageait, fort honnêtement, la plupart des vocations indécises qui venaient implorer sa bénédiction paternelle. Pour qu'il eût consenti à cette exception, il devait avoir des raisons bien fortes. (Le Temps, 9 janvier 1898).

À seulement 23 ans, Rosemonde Gérard publie son premier recueil, Les Pipeaux (1889), qui est immédiatement récompensé par le prix ArchonDespérouses de l'Académie française. C'est à cette époque que la jeune poétesse devient l'épouse d'Edmond Rostand. Sa célébrité l'emporte alors sur celle de son mari. La situation s'inverse néanmoins dès 1898 : le triomphe que remporte Cyrano de Bergerac fait soudain d'Edmond Rostand une gloire nationale. Cette position est encore renforcée par le succès de L'Aiglon en 1900. Au début de l'année 1910, la création de Chantecler occupe une place considérable dans l'actualité littéraire. De nombreux articles sont consacrés à la pièce, très attendue, et à son auteur. Le 15 janvier $1910, \mathrm{Fe}$ mina propose ainsi un diptyque photographique mettant en regard les portraits d'Edmond Rostand et de son épouse.

Le couple de poètes est, visuellement, sur un apparent pied d'égalité : Femina propose deux photographies de même grandeur et pareillement encadrées, de sorte qu'Edmond et Rosemonde semblent également célébrés. Néanmoins, les différents textes d'escorte nuancent cette impression. Rosemonde Gérard est, dans la légende, désignée non sous son nom de jeune fille qui est aussi son nom de plume, mais comme "Madame Edmond Rostand ». Par ailleurs, le court article inséré entre les portraits ne mentionne jamais son activité de poétesse. En somme, Rosemonde figure dans ces

${ }^{1}$ Gaston Deschamps (1861-1931) tient la critique littéraire du Temps à partir de 1893. 
pages non pour ce qu'elle est, mais en raison du lien qui l'unit à Edmond. Elle se trouve ici strictement réduite à son statut d'épouse. Le montage photographique met du reste en scène le couple en tant que tel : les visages, tournés l'un vers l'autre à dessein, donnent le sentiment que mari et femme forment les deux moitiés d'un même tout.

Quelques jours plus tard, Les Annales politiques et littéraires proposent à leurs lecteurs un reportage de sept pages intitulé « Autour d'Edmond
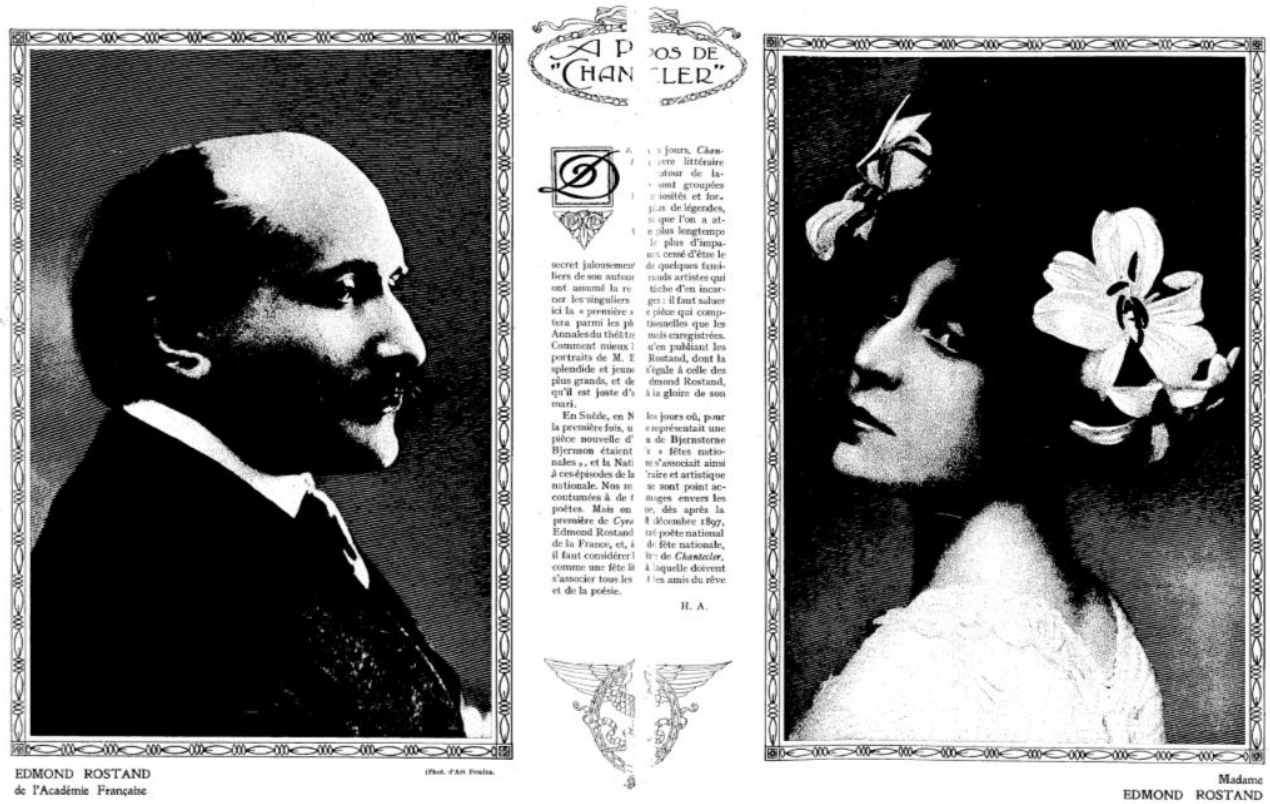

Image 1. Portraits d'Edmond Rostand et Rosemonde Gérard. Femina, 15 janvier 1910.

Rostand »2. L'objectif ainsi poursuivi est d'offrir " un portrait pittoresque et véridique de l'illustre écrivain » (Les Annales politiques et littéraires, 23 janvier 1910). Le reportage est agrémenté de nombreuses photographies représentant non seulement le poète à sa table de travail, mais aussi la villa Arnaga où il réside, ainsi que ses familiers.

Une fois encore, Rosemonde Gérard n'est montrée qu'en raison de son appartenance à la sphère intime du grand homme. Elle est un élément d'apparat parmi d'autres, au même titre que la très belle demeure qu'il a fait construire dans le pays basque (ce que souligne du reste le parallèle établi par la légende de la photographie qui la représente). Jamais il n'est question de ses vers, ni de la reconnaissance à laquelle elle a pu accéder en tant que poétesse. Le traitement que lui réserve la revue est en tout point semblable à celui de la femme de Jean Richepin, qui n'est pourtant pas une autrice3. Compagnes des gloires littéraires du temps, elles ne sont mises en lumière

${ }^{2}$ Le titre est très éloquent : on pressent aussitôt que Rosemonde Gérard ne figurera ici qu'au second plan.

3 Voir, par exemple, la photographie de Jean Richepin accompagné de sa femme et de ses enfants dans Les Annales politiques et littéraires du 26 juin 1910. 
qu'en raison de ce statut, quelles que soient les activités qu'elles puissent mener parallèlement.

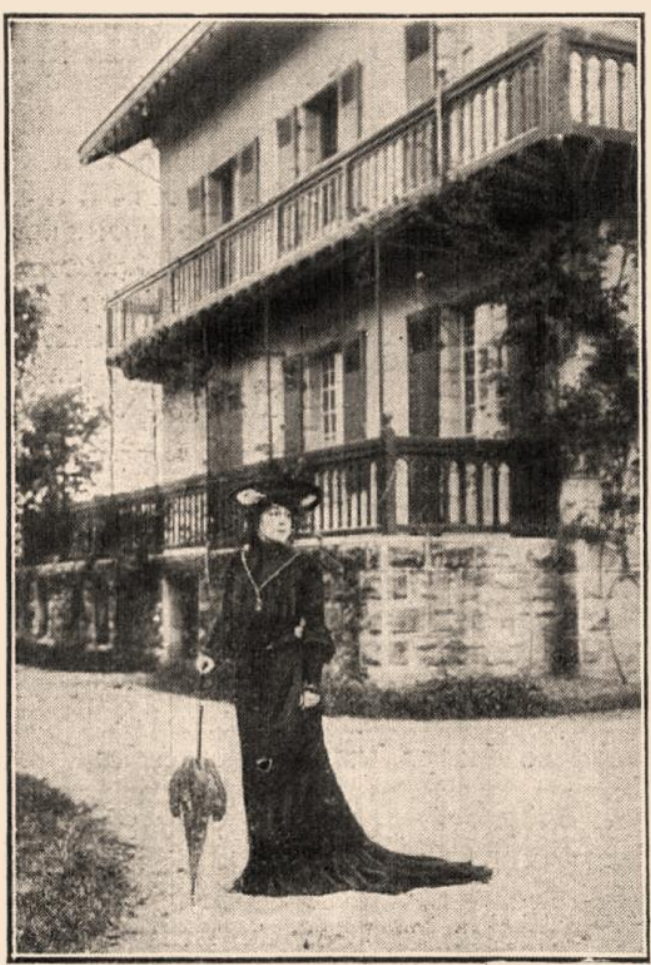

Image 2. Rosemonde Gérard devant la villa Arnaga. Les Annales..., 23 janvier 1910.
La situation de Judith Gautier n'est guère plus enviable. Alors qu'elle vient d'être élue membre de l'Académie Goncourt4 en remplacement de Jules Renard, $\mathrm{Fe}$ mina lui consacre, le 15 novembre 1910, quelques lignes. Dans l'immense portrait photographique qui illustre la nouvelle de son élection, est incrusté un médaillon sur lequel figure le profil de son père, Théophile Gautier.

Ce parallèle visuel inscrit immédiatement la poétesse du Livre de jade (1867) dans une filiation et tend à gommer ses qualités intrinsèques. Ce choix est d'autant plus étonnant qu'à 65 ans Judith Gautier a derrière elle

une œuvre qui pourrait aisément se dispenser du soutien d'une telle figure d'autorité. Récompensée par deux prix de l'Académie française (le prix Alfred Née en 1898 et le prix Maillé-Latour-Landry en 1904), elle s'est alors illustrée dans les trois archigenres. Célébrée lors de la parution de ses deux premiers romans (Le Dragon impérial en 1869 et L'Usurpateur en 1875), la reconnaissance dont elle a joui sur la scène littéraire ne s'est jamais ternie. Dans le corps de l'article qui lui est consacré, Judith Gautier est

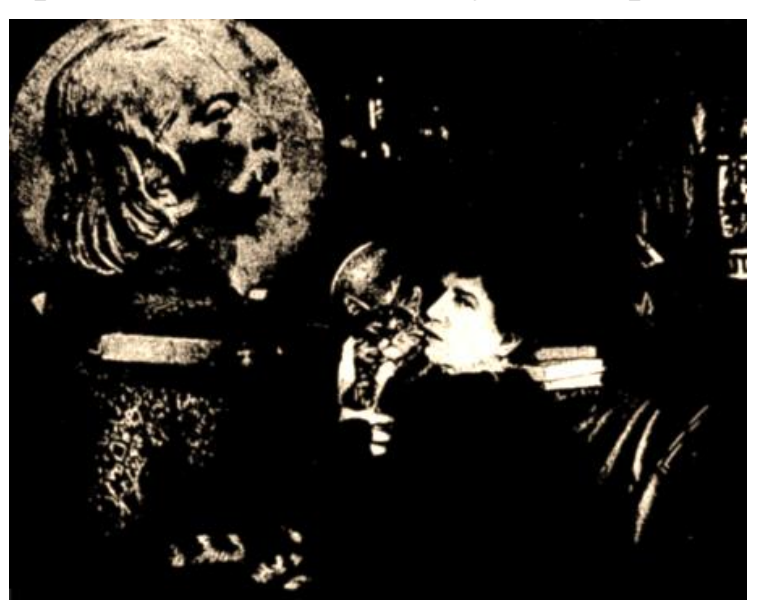

Image 3. Judith Gautier. Femina, 15 novembre 1910. certes célébrée pour son activité littéraire : on la présente comme un « grand

4 Judith Gautier est la première femme à faire partie de l'Académie Goncourt. 
écrivain, qui vit loin des coteries, dans et pour son rêve » (Femina, 15 novembre 1910). Néanmoins, ce geste est contredit par le montage photographique qui la rappelle à son statut familial et la place, symboliquement, sous le joug d'une figure tutélaire masculine. On remarque en effet que le profil de Théophile Gautier domine, par sa taille et par sa position, l'ensemble de l'illustration. La poétesse, située en contrebas, n'attire l'attention du spectateur que dans un second temps. Rattachée à l'univers domestique par le chat qu'elle tient dans les bras, son image s'oppose à celle du grand homme, dont le profil, frappé dans le métal, semble contempler l'avenir - et la postérité.

Le parallèle entre le père et la fille se veut, on l'imagine, élogieux : Théophile Gautier est alors considéré comme l'un des plus grands poètes de son temps. Il est aussi sans doute implicitement motivé par des raisons littéraires, et en particulier par leur goût commun pour la chinoiserie5. Aidée par son précepteur Ding Dunling qui en fit une bonne sinophone, Judith Gautier est entrée en littérature en traduisant dans son Livre de Jade les poésies classiques chinoises de Li Bai, Du Fu ou Wang Bo que son père admirait. De fait, si cette mise sous tutelle peut s'expliquer, elle n'en contribue pas moins à minorer l'exceptionnalité de la poétesse.

Les Annales politiques et littéraires, qui relaient également cette élection inédite, ne font guère mieux. Un portrait photographique de Judith Gautier, d'une remarquable sobriété, illustre la nouvelle.

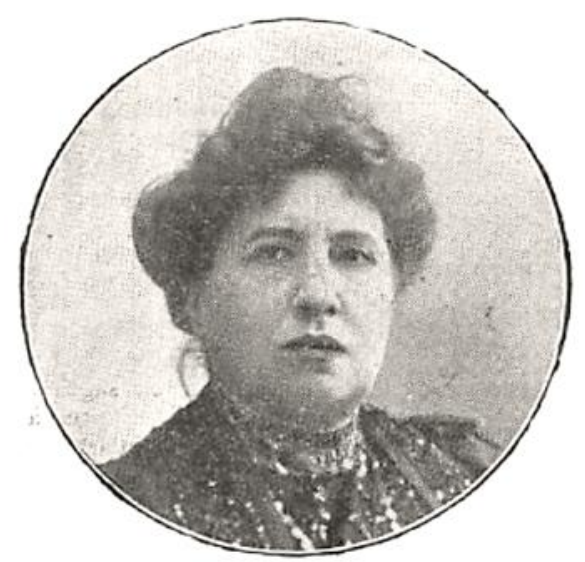

Image 4. $\mathrm{M}^{\text {me }}$ Judith Gautier.

Cette fois, il n'est visuellement pas question de l'inscrire dans une quelconque filiation. Toutefois, le « croquis littéraire » que lui consacre Anatole France ne cesse, quant à lui, de faire allusion aux origines prétendument héréditaires de sa vocation poétique. Dès la première phrase, Judith Gautier y est décrite comme « la fille du poète » (Les Annales politiques et littéraires, 6 novembre 1910). De manière corollaire, loin de lui reconnaître un don inné pour la chose littéraire, Anatole France présente son talent comme le résultat de son éducation. C'est bien parce qu'elle a fréquenté un littérateur de génie qu'elle est, à son tour, devenue une auteure originale. La logique est imparable :

Dans cette petite maison de la rue de Longchamp où, comme il est dit des princesses dans les contes de fées, elle grandissait chaque jour en sagesse et en beauté, Judith apprit, dès l'enfance, à comprendre et à goûter les

5 Tel est le titre d'un poème vraisemblablement écrit par Théophile Gautier en 1835 et publié dans La Comédie de la mort en 1838. 
formes d'art les plus exquises, les plus rares, les plus étranges. Son père, en parlant comme en écrivant, était un incomparable assembleur de merveilles. [...] C'est là qu'enfant Judith Gautier se nourrit de poésie et apprit à aimer la beauté exotique. Pour que son éducation d'artiste fût complète, il ne lui manqua rien, sinon, peutêtre, le commun et l'ordinaire (Les Annales politiques et littéraires, 6 novembre 1910).

On le voit, la tutelle masculine est explicitée tantôt dans l'illustration, tantôt dans le texte d'escorte : nulle issue ne se dessine.

Lorsqu'elles ne sont ni épouses ni « filles de », les poétesses sont tout de même affiliées à un parrain littéraire, garant de la qualité de leur production. À l'occasion de la parution de son recueil, Le Jardin de l'Amour (1910), Lucy Ditte ${ }^{6}$ se voit offrir par Femina un portrait photographique en pleine page. Cette présentation, très prestigieuse, est très vraisemblablement due au titre porté par la poétesse : cette dernière est en effet la veuve du comte George de Pembroke et Montgomery. Les attributs arborés par la poétesse sur son portrait (la canne, les gants, le chien de chasse) sont du reste tout à fait caractéristiques de son statut aristocratique.

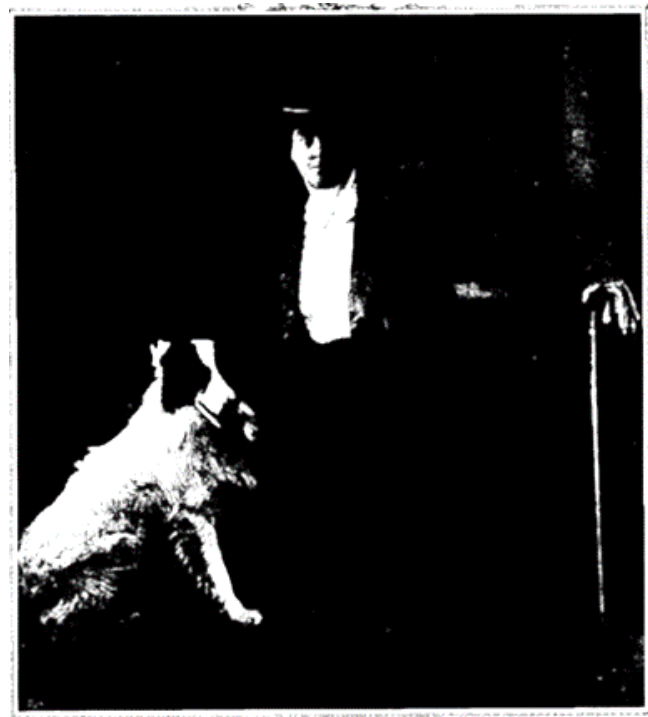

Image 5. $\mathrm{M}^{\text {me }}$ de Montgomery. Femina, 15 mai 1910.

Si Lucy Ditte doit l'honneur qui lui est accordé à la position mondaine de son mari, la légende rappelle, de son côté, qu'elle doit son talent à un grand aîné. On peut en effet y lire que $\mathrm{M}^{\mathrm{me}}$ de Montgomery a été « l'élève du grand maître Sully Prudhomme » (Femina, 15 mai 1910). L'article que lui consacre Max Ligardes insiste du reste sur le fait qu'elle lui " doit beaucoup ». Plus encore, il lui dénie, discrètement, la maternité de son œuvre lorsqu'il soutient que « l'élève fidèle et pieuse a compris que ce recueil où

${ }^{6}$ Lucy Ditte (1861-1926) est une autrice, librettiste et compositrice. 
elle a enclos le plus pur de son cœur et de sa pensée, appartient un peu au grand poète » (Femina, 15 mai 1910).

Si les poétesses ne peuvent échapper à leur condition de fille,

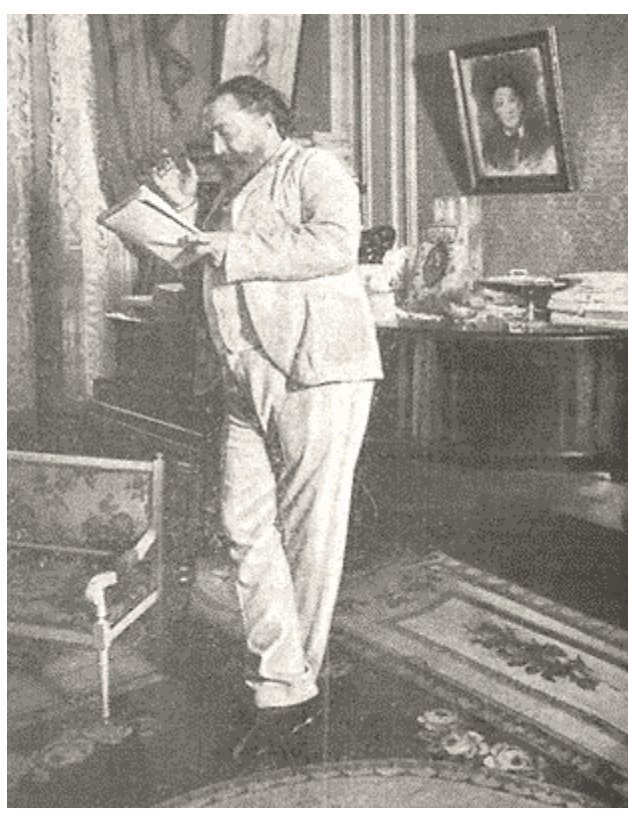

Image 6. Catulle Mendès corrigeant un manuscrit. Les Annales..., 11 novembre 1906. d'épouse ou d'élève, les poètes masculins, quant à eux, sont régulièrement représentés dans leur quotidien de penseurs, sans qu'on les associe nécessairement à leur vie familiale. Les Annales politiques et littéraires photographient ainsi le 11 novembre 1906 Catulle Mendès chez lui, en train de corriger les dernières épreuves de sa nouvelle pièce, La Vierge d'Avila. Nulle mention n'est faite de sa femme, Jane Catulle-Mendès, qui a pourtant publié son premier recueil, Les Charmes, en 1904, et est alors membre du prix «Vie heureuse » depuis deux ans.

De la même manière, en janvier 1910, le journal suit Émile Bergerat, montré à sa table de travail et chez les bouquinistes. Sa parentèle n'est pas davantage évoquée, alors même que son mariage avec Estelle Gautier en fait le gendre de Théophile. 
Çédille, revista de estudios franceses, 20 (2021),

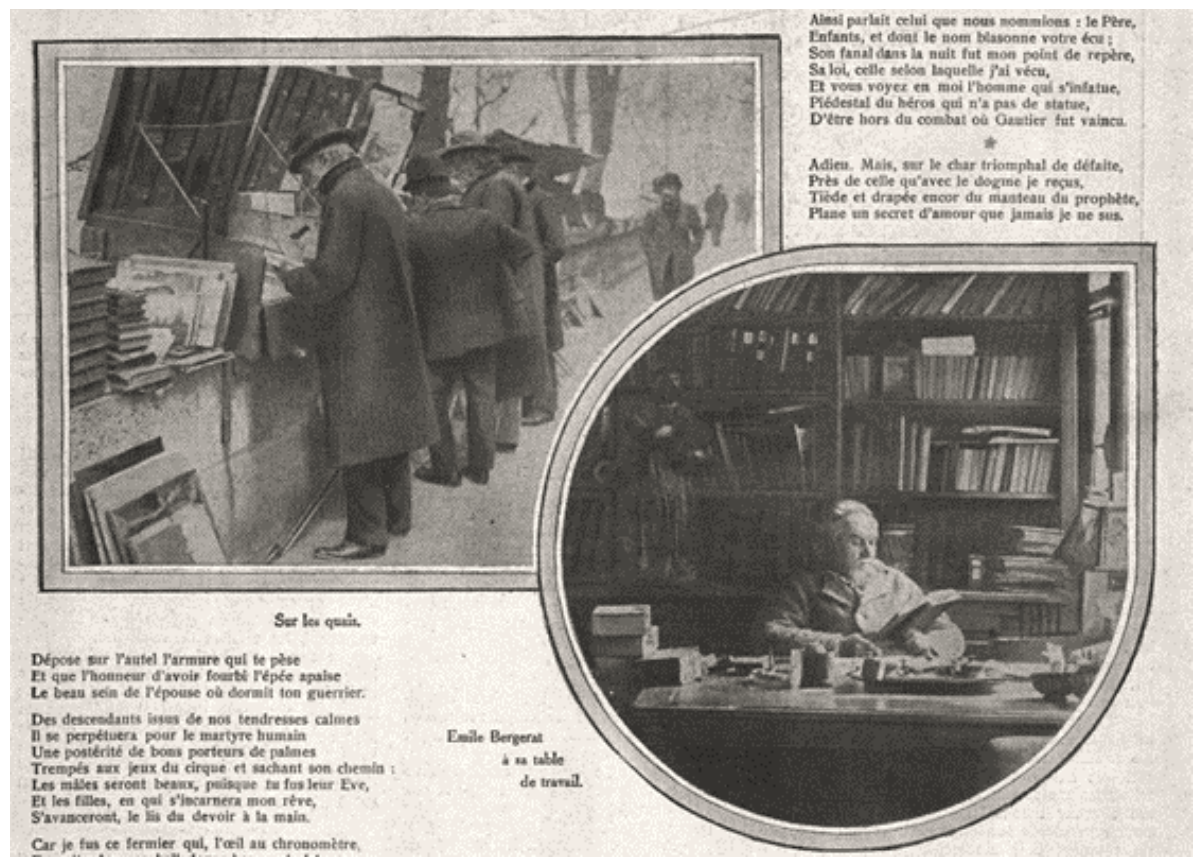

Image 7. Émile Bergerat. Les Annales..., 30 janvier 1910. 
Enfin, contrairement aux poétesses, certains poètes commencent à connaître un état de vedettisation. Si la fin du XIX ${ }^{\mathrm{e}}$ siècle se caractérisait par une " déromantisation » (Diaz, 2007) de la figure auctoriale?, la charnière des XIX ${ }^{\mathrm{e}}$ et $\mathrm{XX}^{\mathrm{e}}$ siècles est l'occasion d'un bouleversement causé par le développement des médias, et en particulier de la presse écrite. Christian Delporte, Claire Blandin et François Robinet l'ont bien décrit (Delporte, Blandin et Robinet, 2016) : les années 1890-1914 signent l'avènement d'une révolution durable. La presse s'industrialise. Surtout, elle se massifie. La lecture cesse d'être collective pour se faire individuelle. Les grands quotidiens sont désormais vendus au numéro, de sorte qu'il n'est plus besoin de s'y abonner pour les recevoir. Le recul de l'analphabétisme produit une démocratisation de la lecture. Enfin, l'image entre dans le journal, au gré d'évolutions techniques successives. Le « reporter », appareil photo portatif, fait son apparition à la fin du XIX ${ }^{\mathrm{e}}$ siècle. Peu à peu, les moyens de reproduction et d'impression s'améliorent, via la similigravure et l'héliogravure. Il devient de plus en plus aisé de diffuser le portrait de celles et ceux qui font l'actualité. Les personnalités littéraires bénéficient, au même titre que les autres, de ces nouveaux moyens de communication qu'il faut apprendre à apprivoiser. L'image fait vendre : elle constitue sans conteste une plus-value. Elle entre pleinement dans les stratégies publicitaires mises en place par les revues pour s'attirer de nouveaux lecteurs. Le cas d'Edmond Rostand est emblématique de cette révolution médiatique: nombreux sont alors les journaux qui tâchent de publier à son sujet des documents iconographiques inédits. Le 6 août 1911, Les Annales proposent ainsi à leurs lecteurs un portrait du poète jeune homme, alors qu'il était encore collégien à Marseille. Signe du degré de célébrité inédit auquel l'au-

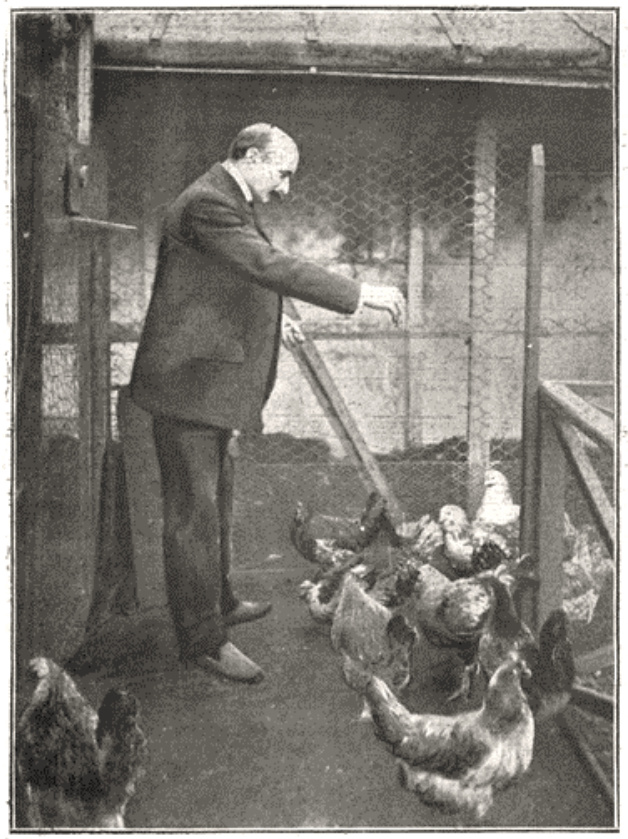

Image 8. « Le sosie d'Edmond Rostand ». Les Annales..., 20 février 1910. teur de Chantecler a accédé, la même revue publie en guise de boutade, le 20 février 1910, une photographie de son sosie, un " aimable journaliste sportif » (Les Annales politiques et littéraires, 20 février 1910), mis en scène dans une basse-cour en train de nourrir des poules. Il faut noter qu'Edmond

7 José-Luis Diaz montre bien que, contrairement à l'époque romantique, à la fin du XIX siècle, les hommes de lettres vont désormais jusqu'à refuser de faire paraître leur portrait en tête de leurs volumes (c'est le cas, par exemple, d'un Flaubert ou d'un Taine). La subjectivation triomphante de la littérature subit un reflux. 
Rostand est la seule personnalité issue du monde littéraire parodiée dans ce numéro des Annales : les autres sosies présentés sont ceux de figures politiques de tout premier plan (le président de la République Armand Fallières, les présidents du Conseil passés ou en exercice Georges Clemenceau et Aristide Briand).

Assez paradoxalement, c'est dans le journal Comodia, quotidien

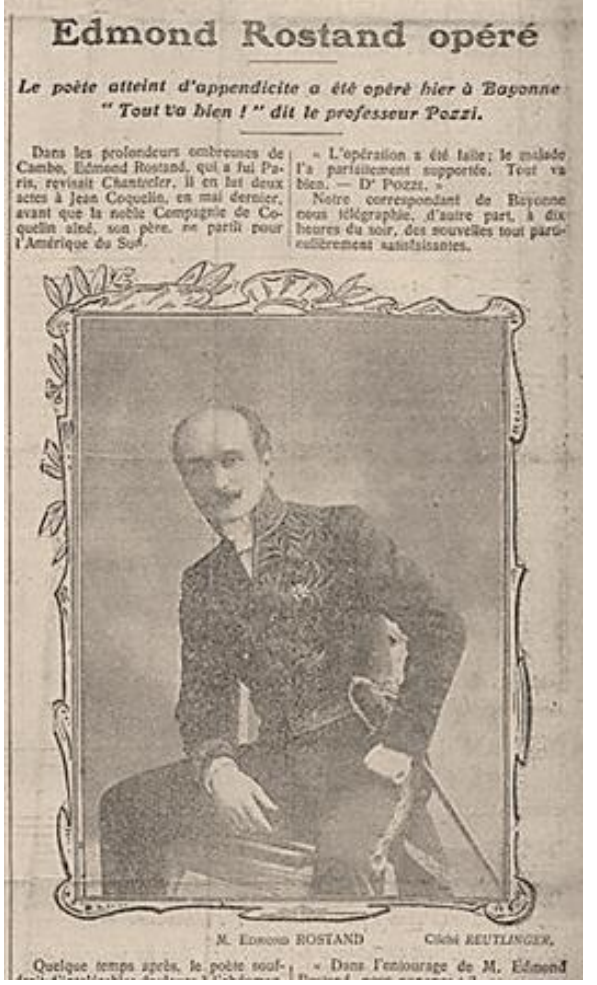

Image 9. Annonce de l'opération d'Edmond Rostand. Comøedia, 6 octobre 1907. d'information culturelle, que l'intérêt pour la vie privée du poète est le plus manifeste. Les portraits officiels d'Edmond Rostand y sont souvent publiés en une afin d'annoncer des événements extra-littéraires comme son opération de l'appendicite réussie (6 octobre 1907) ou ses déplacements entre Paris et Arnaga (10 avril 1909).

Contre toute attente, c'est au public a priori le plus lettré que sont communiquées les informations les plus frivoles.

On le voit, jusqu'en 1910, le déséquilibre de traitement est flagrant entre les poétesses, toujours placées sous une tutelle masculine et ne béné-

ficiant d'une visibilité médiatique qu'en raison de cette aura, et les poètes, reconnus pour leur art et célébrés dans toute leur individualité.

\section{L'émancipation féminine (1911-1914)}

À partir de 1911, les choses changent néanmoins très vite. L'élection de Judith Gautier au sein de l'Académie Goncourt, en dépit de la comparaison obsédante avec son père qu'elle suscite, est un signe de l'évolution des mentalités qui est en train de se jouer. À l'hiver 1910, un grand débat agite l'opinion : il constitue l'autre symptôme de cette évolution. En novembre 1910 en effet, Marie Curie pose sa candidature à l'Académie. Ce geste relance la querelle autour de la présence des femmes sous la Coupole, qui avait déjà été très vive lorsqu'en janvier 1893, Pauline Savari avait eu l'audace de se 
présenter au fauteuil d'Ernest Renan ${ }^{8}$. À partir du 24 novembre 1910, L'Intransigeant lance une enquête afin de désigner les trois femmes les plus aptes à devenir des Immortelles, si toutefois l'Académie consentait à rompre avec la tradition. Les noms d'Anna de Noailles, Gérard d'Houville, Colette ou Judith Gautier sont successivement avancés.

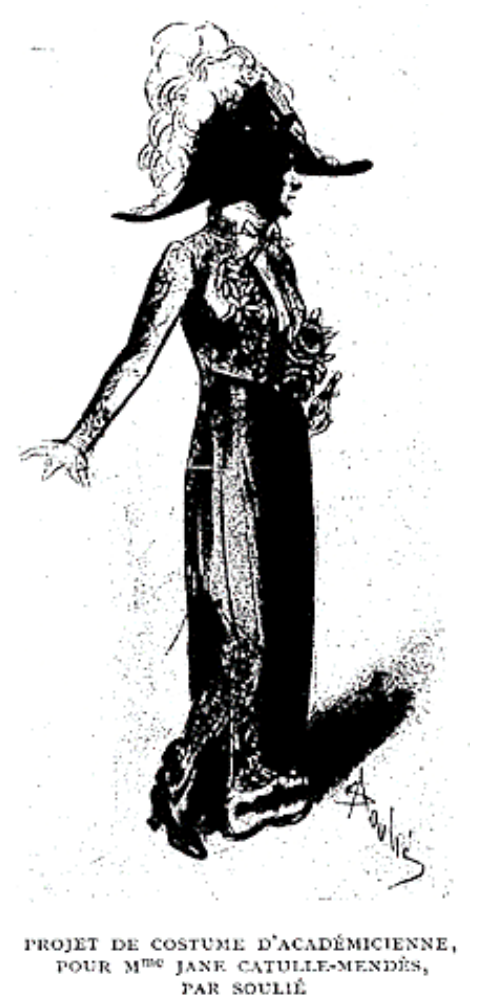

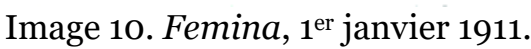

Le $1^{\mathrm{er}}$ janvier 1911, Femina se saisit du sujet et propose aux futures académiciennes des projets de costumes conçus par de grands couturiers comme Paul Poiret ou René Lelong, ou bien encore par des dessinateurs de mode comme A. Soulié.

Les poétesses, sollicitées par la revue, se montrent très prudentes quant à l'issue de l'affaire. En témoigne la réponse avisée de Marcelle Tinayre : « il ne faut pas vendre la peau de l'ours avant de l'avoir tué, ni habiller l'académicienne avant de l'avoir élue » (Femina, 1er janvier 1911). Effectivement, le statu quo prévaudra : la candidature de Marie Curie sera retoquée, et il ne sera plus question de femmes à l'Académie avant longtemps9. Ce débat montre néanmoins que la place des poétesses dans la vie littéraire du temps est en train de changer ${ }^{10}$.

De nouvelles plumes, à l'image d'Alphonse Séché (1909) ${ }^{11}$, Jean de Gourmont (1910) ou Maurice Barrès (1913), prennent fait et cause pour la poésie féminine. Les recensions de recueils publiés par des femmes deviennent plus nombreuses et plus louangeuses. Surtout, elles sont de plus en plus illustrées par le portrait photographique des poétesses, sans que ces dernières soient systématiquement associées à leur statut familial ou à un quelconque maître qui leur aurait tracé la voie. Le 15 juin 1911,

${ }^{8}$ Cette tentative s'était soldée par un échec, au motif que Pauline Savari n'avait pas satisfait à la conscription.

9 Il faudra attendre 1980 et l'élection de Marguerite Yourcenar (c'est-à-dire encore 70 ans) pour voir les choses évoluer.

10 On remarquera que l'évolution est plus lente dans le domaine des lettres que dans celui des arts. Comme le rappellent Christine Bard, Frédérique El Amrani et Bibia Pavard, l'École des Beaux-Arts devient mixte dès 1897 et, en 1903, un décret autorise les candidatures féminines au Prix de Rome. Voir leur Histoire des femmes dans la France des XIX et XX ${ }^{e}$ siècles, Paris, Ellipses, 2013.

${ }^{11}$ Son ouvrage, Les Muses françaises, reçoit le prix de la critique décerné par l'Association des Critiques littéraires en janvier 1910. Les Annales politiques et littéraires s'en font l'écho le 9 janvier 1910. 
Femina propose ainsi un portrait-médaillon de Jeanne Dortzal afin d'agrémenter l'article que Henri Duvernois consacre à son recueil Sur les toits bleus du soir. De la même façon, la longue série qu'Auguste Dorchain consacre aux femmes poètes dans Les Annales en 1913 est accompagnée de photographies destinées à faire connaître au public le visage des poétesses évoquées.

Suivant la même stratégie publicitaire, Femina publie le 15 avril 1911, à l'occasion de la huitième fête de la poésie féminine organisée par le journal, la photographie de l'ensemble des lauréates primées depuis la création de l'événement.

LA DUCHESSE DE ROHAN CÉLĖBRE LES LAURÉATES DE FEMINA

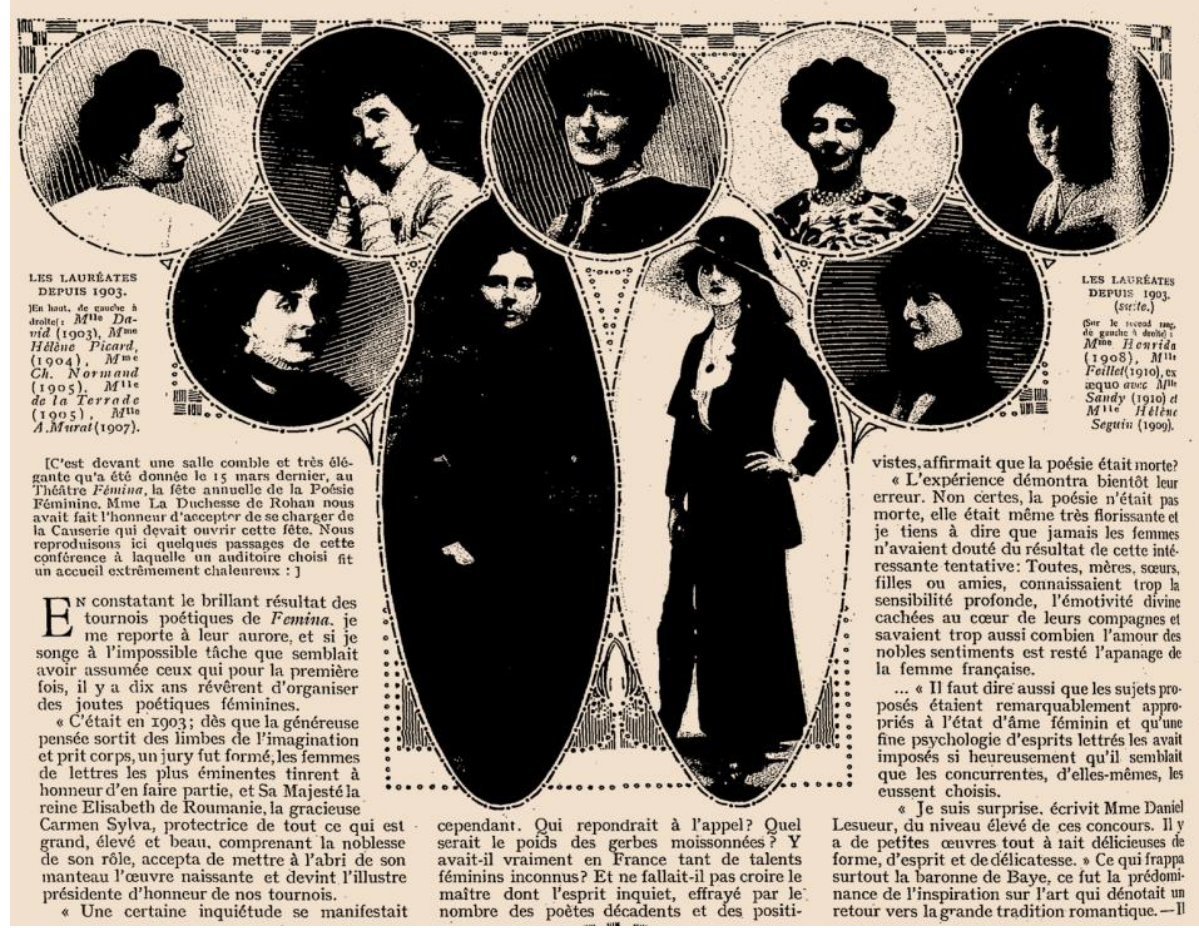

Image 12. Les lauréates du prix de poésie. Femina, 15 avril 1911.

Jusqu'alors, seules les poétesses mondaines et parisiennes jouissant d'une reconnaissance en raison de la protection que leur accordait un homme (qu'il soit leur père, leur époux, ou leur maître littéraire) pouvaient prétendre à l'honneur de voir leur portrait reproduit dans la presse ${ }^{12}$. Désormais, de jeunes inconnues, souvent provinciales, accèdent à cette forme de légitimation. C'est le cas, par exemple, d'Isabelle Sandy ${ }^{13}$, poète lauréate

${ }^{12}$ L'exemple de Rosemonde Gérard, Judith Gautier et $\mathrm{M}^{\text {me }}$ George de Montgomery le montre assez.

${ }^{13}$ Isabelle Sandy (pseudonyme d'Isabelle Fourcade) est née en 1884 à Cos dans l'Ariège. En 1910, elle n'a encore publié aucun recueil. Elle ne bénéficie d'aucun parrainage dans le mi- 
du prix Femina en 1910, dont le visage est révélé par la revue, au milieu de ceux, plus largement diffusés, d'Hélène Picard ${ }^{14}$ ou Hélène Seguin ${ }^{15}$.

Émancipées de la tutelle masculine qui pesait sur elles, les poétesses connaissent à leur tour, dans l'immédiat avant-guerre, un début de vedettisation. Femina recueille ainsi, le $1^{\mathrm{er}}$ août 1914, l'avis de " quelques-unes des plus notoires, voire des plus célèbres Parisiennes de notre temps » sur leur rapport à la mer. Parmi les huit femmes interrogées figurent trois poétesses (Anna de Noailles, Gérard d'Houville et Lucie Delarue-Mardrus), trois actrices (Marcelle Géniat, Sarah Bernhardt et Réjane), une romancière (Myriam Harry) et une danseuse (Natacha Trouhanova). Les femmes de lettres sont ici considérées comme des personnalités à part entière, au même titre que des artistes issues du monde du spectacle. La manière de les représenter est du reste très similaire. L'article est agrémenté d'une photographie en pleine page d'Anna de Noailles

en villégiature en Sicile. La comtesse pose dans les ruines d'Agrigente, le regard tourné vers l'objectif, dans une mise en scène très étudiée.

lieu des lettres. Son statut est bien celui d'une parfaite inconnue. La reconnaissance n'adviendra pour elle qu'à la fin des années 1910, avec l'attribution du Prix Montyon pour son premier roman, Chantal Daunoy.

14 Hélène Picard, née en 1873 , bénéficie déjà d'une belle notoriété : elle a été primée aux Jeux floraux de 1898 et 1900, et a reçu le prix Archon-Despérouses en 1907.

${ }^{15}$ Hélène Seguin a quant à elle été révélée un an plus tôt, en 1910, avec Le Réseau fragile, qui a reçu à la fois le prix Femina et le prix Archon-Despérouses. 


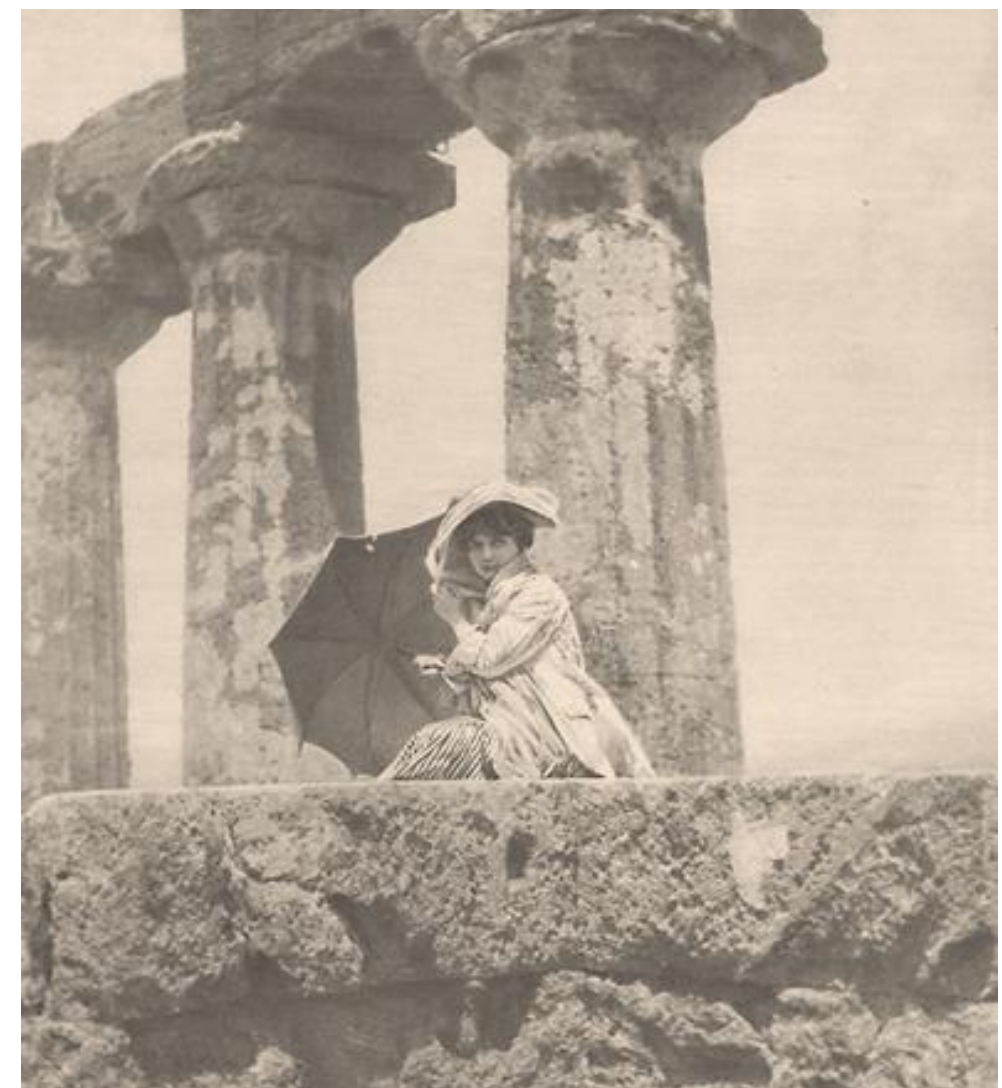

Image 13. Anna de Noailles en villégiature. Femina, $1^{\text {er }}$ août 1914.

La position de la poétesse (la torsion du corps, destinée à donner du mouvement à l'image fixe) fait bien sûr songer aux attitudes de Sarah Bernhardt qui s'était fait une spécialité de ces sinuosités serpentines. L'actrice est elle aussi saisie dans son intimité par la revue, qui la montre dans son refuge de Belle-île.

Littératrices et actrices sont désormais également dignes de figurer dans un reportage photographique : la réalité de leur vie privée devient un sujet de curiosité. 


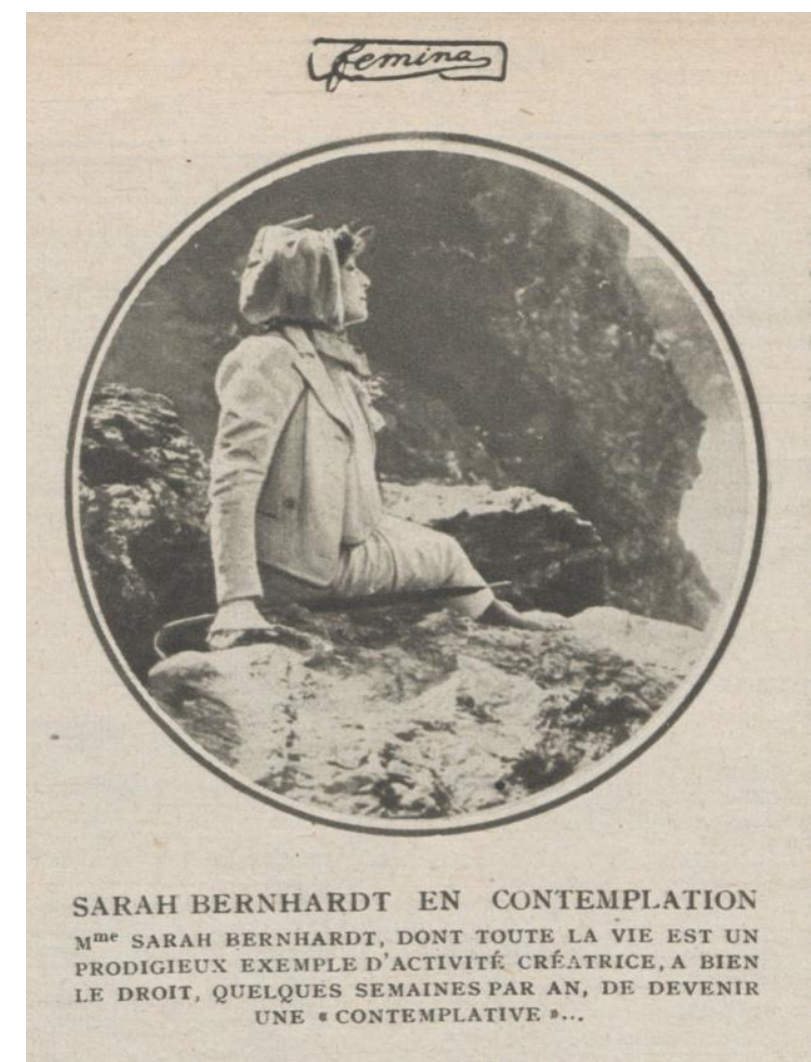

Image 14. Sarah Bernhardt à Belle-Île, Femina, $1^{\text {er }}$ août 1914.

La célébrité nouvelle à laquelle accèdent les femmes poètes a immanquablement son revers. Les Annales se font par exemple l'écho de l'émoi que provoquent, à l'été 1913, les caricatures d'André Rouveyre.

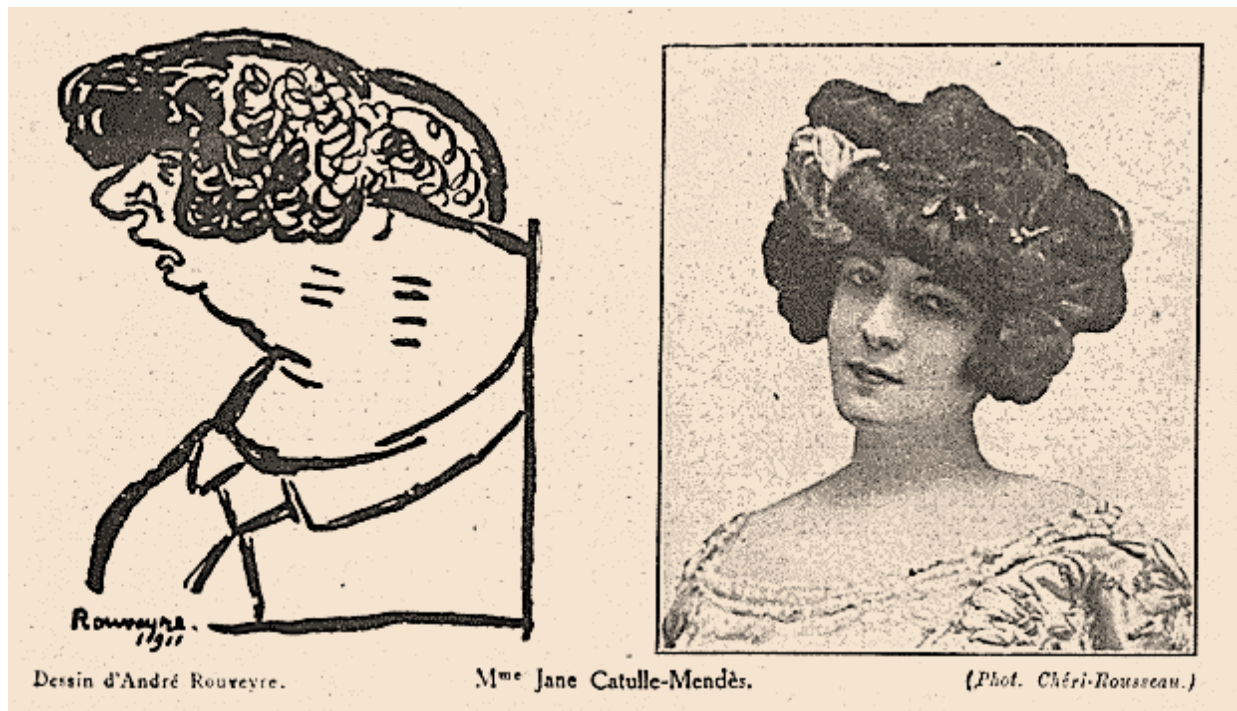

Image 15. Jane Catulle-Mendès caricaturée par André Rouveyre. Les Annales..., 22 juin 1913. 
Cible du dessinateur, Jane Catulle-Mendès (6 juillet 1913) proteste énergiquement contre ce qu'elle juge être non seulement de la « diffamation » mais encore une " injure », tandis que les lecteurs, dont la revue a sollicité l'avis, ne voient dans cet épisode que la rançon de la gloire. Pour la poétesse, la « malveillance », "l'outrage physique et moral » ne sauraient se justifier en aucune manière : " la vraie rançon d'une renommée de bon aloi, c'est le travail sans répit ». Cette réaction souligne bien le déplacement qui s'est peu à peu opéré. Jane Catulle-Mendès est ici moquée non pour son œuvre, comme ce pouvait encore être le cas en 1904 au moment de la parution des Charmes, mais bien pour son apparence physique : elle est entretemps devenue une personnalité publique que les caricaturistes peuvent croquer (avec plus ou moins de bienveillance) en sachant qu'elle sera reconnue par les lecteurs.

À la veille de la guerre la représentation des poètes et des poétesses est désormais sensiblement la même. Dès 1913, les femmes poètes sont enfin montrées pour ce qu'elles sont : des autrices à part entière, en proie aux heurs et malheurs de la médiatisation. Plusieurs facteurs sont susceptibles d'expliquer cette lente reconnaissance. Il faut d'abord mentionner le progrès de l'éducation des femmes (Mayeur, 2008 [1979]). Comme le remarquent Christine Bard, Frédérique El Amrani et Bibia Pavard (2013 : 111), « les femmes gagnent en autonomie en accédant à l'instruction ». Or, la charnière des $\mathrm{XIX}^{\mathrm{e}}$ et $\mathrm{XX}^{\mathrm{e}}$ siècles constitue un véritable tournant. Dès 1880 , les lycées publics de jeunes filles sont créés avec la loi Camille Sée : leur fréquentation s'accentue dans les années 1900. Pour développer ces lycées, il faut procéder au recrutement d'un personnel enseignant féminin. C'est la raison pour laquelle l'École normale supérieure de Sèvres est fondée en 1881. L'apprentissage du latin, matière jusque-là réservée aux garçons, est instauré dans les lycées et collèges féminins en 1913. Le nombre de femmes accédant à l'université quadruple entre 1900 et $1914^{16}$ (Winock, 2020). À la veille de la Première Guerre mondiale, les premières thèses de doctorat en lettres et en philosophie écrites par des femmes sont soutenues ${ }^{17}$. Il est désormais impossible d'exclure de la vie intellectuelle du temps celles qui contribuent activement à l'animer. Ce développement qualitatif et quantitatif de l'éducation des femmes prend place au moment où la France commence à entrer en « régime médiatique » (Kalifa, 2001 : 109). L’imprimé connaît une révolution, la lecture se démocratise, les illustrations fleurissent. Les produits cul-

${ }^{16}$ La première jeune fille admise à l'École normale supérieure de la rue d'Ulm, M ${ }^{\text {lle }}$ Rouvière, l'est en 1910. La première agrégée de grammaire, $M^{\text {lle }}$ Raison, est reçue en 1912.

${ }_{17} \mathrm{M}^{\text {lle }}$ Duporal est reçue docteure ès-lettres avec la mention très honorable et $\mathrm{M}^{\text {lle }}$ Zantal devient docteure en philosophie. Comme le remarque Carole Christen-Lécuyer (2000), le doctorat d'État ès-lettres est celui que les femmes conquièrent en dernier (après la médecine, les sciences, le droit et la pharmacie). 
turels deviennent, peu à peu, des marchandises. La production littéraire féminine s'intègre, au même titre que celle des hommes, à cette culture de masse émergeante.

\section{Conclusion}

Au-delà de l'évolution que nous avons retracée, notre rapide coup de sonde dans la presse de la fin de la Belle Époque ne manque pas de faire surgir un certain nombre de considérations de nature plus synchroniques. Tout d'abord, il est frappant de constater que Femina, revue destinée à un lectorat féminin, ne se distingue en rien de journaux plus généralistes comme Les Annales ou Comodia pour ce qui touche à la représentation des poétesses. Jusqu'en 1911, l'insistance sur le parrainage masculin dont elles bénéficient est la même. Comøedia se distingue sur au moins trois points. Paradoxalement, ce quotidien d'actualité littéraire est celui qui résiste le plus à la représentation des femmes poètes. Entre 1908 et 1914 fort peu de photographies de poétesses y sont publiées ${ }^{18}$. Deuxième spécificité, corollaire de la première : dans ce journal, la variété des clichés reproduits est extrêmement faible, comparativement aux deux autres titres que nous avons étudiés. Les mêmes portraits sont inlassablement réemployés, quelle que soit la nature de l'information qui y est associée. Enfin, Comødia penche, plus encore que Femina ou Les Annales, vers une vedettisation des personnalités littéraires. Le journal tend du reste à se polariser sur Edmond Rostand, qu'il contribue à élever au rang de poète national. Le déséquilibre est très net, entre les photographies de l'auteur de Chantecler, innombrables, et celles d'autres auteurs, beaucoup plus rares. Pour finir, on remarque que, quels que soient l'époque ou le média concernés, la presse aime à montrer les poètes dans leur intimité et à livrer aux lecteurs des détails biographiques à leur sujet. Or, cette curiosité ne s'applique pas encore avec la même ardeur aux poétesses : il faudra pour cela attendre la fin des années 1920 et l'apparition des magazines photographiques.

\section{RÉFÉRENCES BIBLIOGRAPHIQUES}

BARD, Christine, Frédérique El AMrani \& Bibia PAVARD (2013): Histoire des femmes dans la France des XIX ${ }^{e}$ et $X X^{e}$ siècles. Paris, Ellipses.

BARRÈs, Maurice (1913) : «Mme Mathieu de Noailles ». Les Annales politiques et littéraires, 6 juillet.

CATUlLE-MEndÈs, Jane (1904) : Les Charmes. Paris, Fasquelle.

CATUlle-MEndÈs, Jane (1913) : «Lettre à André Rouveyre ». Les Annales politiques et littéraires, 6 juillet.

${ }^{18}$ Nous n'avons trouvé, en l'état actuel de nos recherches, qu'un portrait de $\mathrm{M}^{\mathrm{me}}$ AlphonseDaudet dans le numéro de Comøedia daté du 5 mars 1912. 
CHRISTEN-LÉCUYER, Carole (2000) : « Les premières étudiantes de l'Université de Paris ». Travail, genre et sociétés, 4, 35-50.

DÉCAUDIN, Michel (2001) : La Crise des valeurs symbolistes. Vingt ans de poésie française, 1895-1914. Genève, Slatkine Reprints.

DELPORTE, Christian, Claire BLANDIN \& François RoBINET [dir.] (2016) : Histoire de la presse en France, $X X^{e}-X X I^{e}$ siècles. Paris, Armand Colin.

DIAZ, José-Luis (2007) : L'Écrivain imaginaire. Paris, Champion.

DORTZAL, Jeanne (1911) : Sur les toits bleus du soir. Paris, À la Belle Édition.

FERNAND GREGH, Harlette (1907) : Jeunesse. Paris, Sansot et Cie.

GAUTIER, Judith (1867) : Le Livre de jade. Paris, Lemerre.

GÉRARD, Rosemonde (1889) : Les Pipeaux. Paris, Fasquelle.

Gourmont, Jean de (1910) : Muses d'aujourd'hui. Essai de physiologie poétique. Paris, Mercure de France.

Holmes, Diana \& Carrie TARR [dir.] (2006) : A «Belle Epoque »? Women in French Society and Culture, 1890-1914. New York-Oxford, Berghahn Books.

IZQUIERDO, Patricia (2009) : Devenir poétesse à la Belle Époque (19oo-1914). Étude littéraire, historique et sociologique. Paris, L'Harmattan.

Kalifa, Dominique (2001) : La Culture de masse en France. Tome 1, 1860-1930. Paris, La Découverte.

LE Guennec, François \& Nicholas-Henri ZMelty (2013) : La Belle Époque des femmes ? Paris, L'Harmattan.

MAYEUR, Françoise (2008) : L'Éducation des filles en France au XIX $X^{e}$ siècle. Paris, Perrin (coll. Tempus).

NoAILLES, Anna de (1907) : Les Éblouissements. Paris, Calmann-Lévy.

PICARD, Hélène (1907) : L'Instant éternel. Paris, Sansot et Cie.

Pluet-Despatin, Jacqueline, Michel LEYMARIE, \& Jean-Yves Mollier [dir.] (2002) : La Belle Époque des revues (1880-1914). Paris, Éditions de l'Institut Mémoires de l'édition contemporaine.

PRIN-CONTI, Wendy (2019) : Femmes poètes de la Belle Époque : heurs et malheurs d'un héritage. Paris, Champion.

PRIN-CONTI, Wendy (à paraître) : « La représentation des femmes poètes dans Les Annales politiques et littéraires (1900-1914) », in A. Auzoux, C. Koskas et E. Rousso, Actes du colloque "Des revues et des femmes », organisé les 28 et 29 mai 2019 à l'Université Paris Sorbonne. À paraître chez Champion (coll. Littérature et genre).

SÉcHÉ, Alphonse (1909) : Les Muses françaises. Paris, Louis-Michaud.

WinOCK, Michel (2002) : La Belle Époque, La France de 19oo à 1914. Paris, Perrin. 